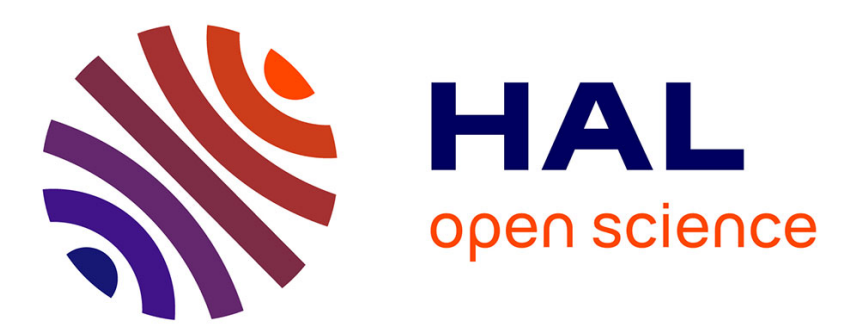

\title{
On noise reduction in strain maps obtained with the grid method by averaging images affected by vibrations
}

\author{
Frédéric Sur, Michel Grediac
}

\section{To cite this version:}

Frédéric Sur, Michel Grediac. On noise reduction in strain maps obtained with the grid method by averaging images affected by vibrations. Optics and Lasers in Engineering, 2015, 66, pp.210-222. 10.1016/j.optlaseng.2014.09.013 . hal-01075764

\section{HAL Id: hal-01075764 \\ https://inria.hal.science/hal-01075764}

Submitted on 20 Oct 2014

HAL is a multi-disciplinary open access archive for the deposit and dissemination of scientific research documents, whether they are published or not. The documents may come from teaching and research institutions in France or abroad, or from public or private research centers.
L'archive ouverte pluridisciplinaire HAL, est destinée au dépôt et à la diffusion de documents scientifiques de niveau recherche, publiés ou non, émanant des établissements d'enseignement et de recherche français ou étrangers, des laboratoires publics ou privés. 


\title{
On noise reduction in strain maps obtained with the grid method by averaging images affected by vibrations
}

\author{
Frédéric SuR* and Michel GRÉDiaC ${ }^{\dagger}$
}

\begin{abstract}
Any image-based contactless measurement system has a limited resolution because of sensor noise. If the sensor is rigorously static with respect to the imaged object, a possibility is to reduce noise by averaging images acquired at different times. This paper discusses images of a pseudo-periodic grid used in experimental solid mechanics to give estimations of in-plane displacement and strain components of a deformed flat specimen. Because of the magnification factor which is employed, the grid images are often affected by residual vibrations, thereby invalidating the assumption that the sensor is static. The averaged grid image is thus a biased estimator of the unknown noise-free image. In spite of this, we prove that the retrieved displacement and strain components still benefit from noise reduction by time-averaging. A theoretical model is discussed, and experiments on real and synthetic data sets are provided.
\end{abstract}

Keywords: Strain map denoising, residual vibrations, grid method, signal-dependent sensor noise.

This is the accepted version of the following article:

F. Sur and M. Grédiac. On noise reduction in strain maps obtained with the grid method by averaging images affected by vibrations. Optics and Lasers in Engineering, vol. 66, p. 210-222, Elsevier, 2015.

DOI: $10.1016 / j$.optlaseng. 2014.09.013

*LORIA, UMR CNRS 7503, Université de Lorraine, CNRS, INRIA project-team Magrit; Campus Scientifique, BP 239, 54506 Vandœuvre-lès-Nancy Cedex, France.

${ }^{\dagger}$ Institut Pascal, UMR CNRS 6602, Université Blaise Pascal, CNRS; BP 10448, 63000 Clermont-Ferrand, France. 


\section{Introduction}

Full-field measurement techniques are now wide spread in the experimental mechanics community, but characterizing their actual metrological performance still remains an open question, as illustrated by numerous papers published recently on this topic $[3,5,6,10,15,29]$. In particular, it is important to understand all phenomena which cause the final displacement and strain maps to be noisy or affected by a bias in order to tackle these causes with suitable tools, and finally obtain more reliable maps. In this context, this paper is devoted to the effect of vibrations on time averaging which is often used to reduce noise in images before processing them to obtain displacement and strain maps. It is shown that, under vibrations, an averaged image is actually a biased estimator of the noise-free image. Such a property makes it necessary, in standard image processing applications, to accurately estimate the translation affecting each image in order to properly register the images before stacking. However, the contribution of this paper is to demonstrate that, in the special case of the grid method, the displacement and strain maps estimated from the averaged grid are still improved by this averaging procedure and the registration step is not needed.

The grid method is one of the full-field techniques available for measuring in-plane displacement and strain maps of a specimen subjected to a load, and consequently locally slightly deformed [25]. It first consists in depositing a regular grid on the surface of the flat specimen to be tested and then in taking high-resolution images of the grid before and after deformation, see Figure 1 (left). Since the experimental setup makes it possible to precisely align the imaged grid with the pixel grid, a grid image is modeled as a 2-D pseudo-periodic function [4, 11, 23]:

$$
s(x, y)=\frac{A}{2}\left(2+\gamma \ell\left(2 \pi f x+\phi_{1}(x, y)\right)+\gamma \ell\left(2 \pi f y+\phi_{2}(x, y)\right)\right)
$$

where $A>0$ is the average field illumination, $\gamma \in[0,1]$ is the contrast of the oscillatory pattern, the line profile $\ell$ is a $2 \pi$-periodic real function with peak-to-peak amplitude equal to 1 and average value 0 , $f$ is the frequency of the carrier (e.g., $f=1 / 5$ pixel $^{-1}$ in Figure 1$)$, and $\phi_{1}(x, y)$ and $\phi_{2}(x, y)$ are the carrier phase modulations along the $x$ - and $y$-axes.

Before deformation, $\phi_{1}$ and $\phi_{2}$ model the deviation from ideality of the manufactured grid. After deformation, the phase modulations are caused by this deviation and to local surface displacements brought by deformation. The displacements along the $x$ - and $y$-axes are actually proportional to $\Delta \phi_{1}$ and $\Delta \phi_{2}$ respectively, and the linearized strain components are linear combinations of $\Delta \partial \phi_{1} / \partial x, \Delta \partial \phi_{1} / \partial y, \Delta \partial \phi_{2} / \partial x$, and $\Delta \partial \phi_{2} / \partial y$, where $\Delta$ is the difference between the maps before and after deformation [2]. It is important to note that the derivatives of $\phi_{1}$ and $\phi_{2}$ are very small with respect to $2 \pi f$ for most constitutive materials used in real structures $\left(10^{-2}-10^{-4}\right.$ vs. $2 \pi / 5$ pixel $\left.^{-1}\right)$. This property allows us to go beyond standard approaches based on Fourier transform to analyze grids used in, e.g., fringe pattern analysis in optical interferometry [13, 25].

A major cause of measurement uncertainty is sensor noise. The uncertainty on the phases and their derivatives has been quantified in the case of the classic windowed Fourier estimation of the phase in [23] after [24] for an ideal Gaussian noise (cf. [20] for a short presentation) and in the case of displacement and strain components in [10] for a realistic Poisson-Gaussian noise. Reducing the uncertainty could be achieved by reducing first the noise level in the grid images. It is not possible to use off-the-shelf denoising algorithms from the image processing literature (see, e.g., the recent review papers $[16,17]$ ) since the aim here is to obtain a guaranteed measurement of tiny quantities instead of a pleasingto-the-eye output. A simpler, well-founded approach would be to average a series of $T$ views of the fixed grid. The intensity measured at a given pixel being made of the "true" intensity added to an independent noise, this average would be a consistent unbiased estimation of the true intensity, within a $\mathcal{O}(1 / \sqrt{T})$ confidence interval. However, the experimental setting requires large magnification factors. 

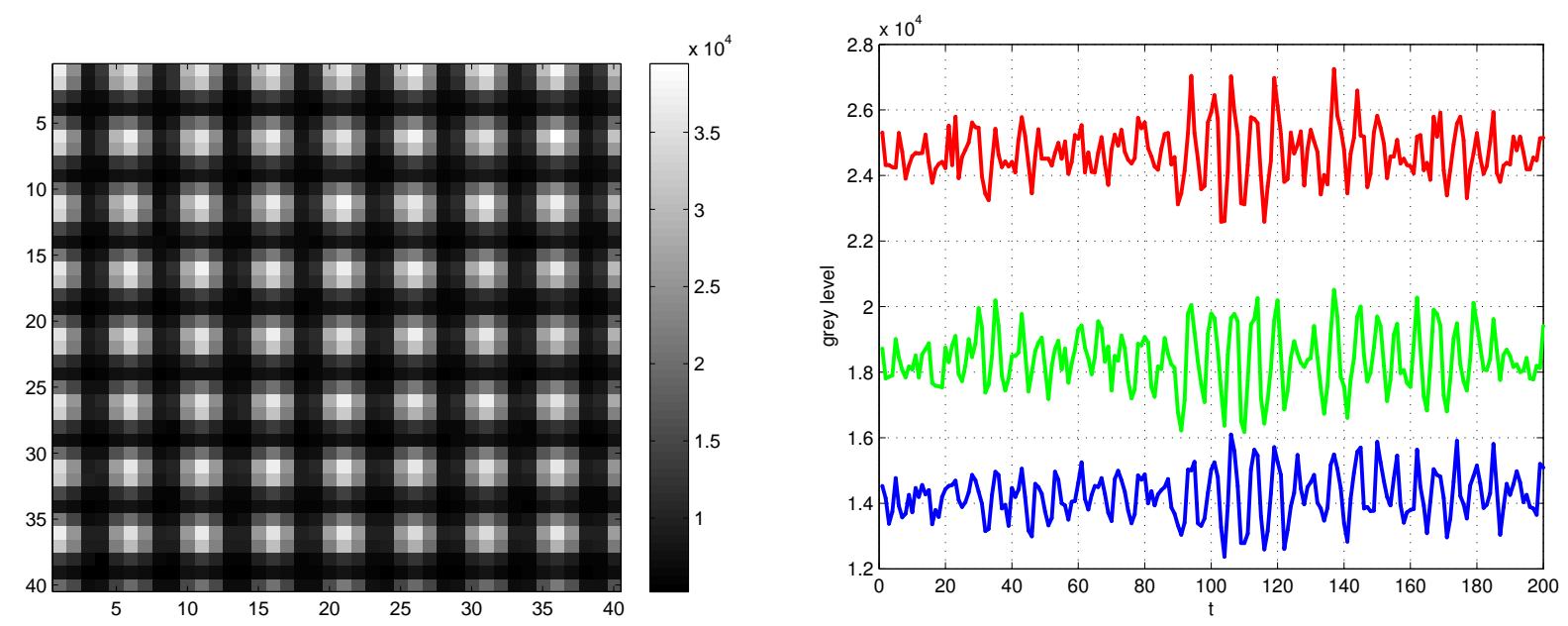

Figure 1: Left: Close-up of the grid on a deformed specimen. In this typical example, the deformations are not visible to the naked eye. Right: Typical time evolution of the gray level of three distant pixels lying between lines of the grid. The in-phase oscillations are due to mechanical vibrations and not to the sensor noise. Note that the output of the 12-bit Sensicam QE camera which is used here is natively encoded in a 16-bit image by simply multiplying the gray levels by $2^{4}$, which explains the range of the pixel intensities.

For example, in Figure 1 (left) the side of each pixel measures 40 micrometers on the specimen. Even though the camera and the testing machine are carefully installed and fixed, residual vibrations generally occur, as depicted in Figure 1 (right). This is due to the fact that experiments are generally carried out in practice with testing machines directly resting on concrete slabs, not on optical tables. Even though this does not correspond to harsh experimental conditions, micro-vibrations borne by slabs generally induce slight variations from one image shot by the camera to another. These variations are added to the noise due to camera sensor. Consequently, there are legitimate questions on the impact of this additional impairment on the image averaging procedure, which is commonly performed to decrease the impact of sensor noise on images and is a built-in function of some cameras [1]. As a consequence of these residual vibrations, it will be shown that the average pixel intensity does not converge to the true underlying intensity, contrary to the perfectly static case.

The contribution of this paper is to prove that the averaged grid image is actually a biased estimator of the noise-free grid (this property being general and not specific to grid images), but that the phases and phase derivatives estimated from the averaged grid are still improved by this averaging procedure. We also show that estimating these quantities from each grid image separately and averaging afterwards basically gives the same result, though this latter case is much more computationally intensive. It is noteworthy that these simple procedures do not need any estimation of the vibration amplitude affecting each image, contrary to most existing stacking or super-resolution approaches (see [27] for an example in thermographic imaging). Let us also mention that we assume here that the exposure time is short enough so that no motion blur can be observed in the imaged grids, which is realistic in practical experiments since the light source is sufficiently powerful to permit short exposures.

The paper is organized as follows. Section 2 discusses the theoretical model. Section 3 confirms the theory on real data and on synthetic data for a sanity check. We conclude with Section 4. 


\section{Noise reduction by image averaging in the presence of vibrations}

Let us consider a series of $T$ images impaired by vibrations. The gray level of a pixel $(x, y)$ in the $t$-th image can be modeled by

$$
v(x, y, t)=s\left(x+\alpha_{t}, y+\beta_{t}\right)+n_{s\left(x+\alpha_{t}, y+\beta_{t}\right)}(x, y, t)
$$

where

- $s(x, y)$ is the unknown noise- and vibration-free reference image.

- $\left(\alpha_{t}, \beta_{t}\right)$ is the unknown displacement translation vector due to vibrations between the reference image and the $t$-th image. It is an independently identically distributed 2-D 0-mean random process.

- $n_{l}(x, y, t)$ is a random variable modeling an additive signal-dependent noise, independently distributed in $x, y, t$, such that its expectation is 0 and its variance is a linear function of the gray level $l$. For instance, this model is valid for the Poisson-Gaussian noise affecting linear CMOS or CCD sensors $[7,8,9,12]$ which are used in practice [4].

The model of (2) was used in [22] (see also [21]) for sensor noise measurement from a series of grid images affected by vibrations.

Let us assume that the amplitude of the vibrations is small enough so that it is justified to identify $s\left(x+\alpha_{t}, y+\beta_{t}\right)$ and its second-order Taylor expansion such that

$$
s\left(x+\alpha_{t}, y+\beta_{t}\right)=s(x, y)+\left(\alpha_{t}, \beta_{t}\right) \nabla s(x, y)+\frac{1}{2}\left(\alpha_{t}, \beta_{t}\right) H_{s}(x, y)\left(\alpha_{t}, \beta_{t}\right)^{T}
$$

where $\nabla s$ is the gradient and $H_{s}$ is the Hessian matrix of $s$. Note that the model in $[21,22]$ just needs a simpler first-order expansion which makes the subsequent calculations easier. The reason behind this is that these latter papers do not require accurate local estimations but rest on spatial averages which discard the effect of the Hessian in (3).

The next section discusses the grid image obtained by averaging the series of the $T$ images affected by vibrations.

\subsection{Effect of vibrations on the averaged image}

In the remainder of this paper, the standard deviation (resp. variance) of any random variable $X$ is denoted by $\operatorname{Std}(X)$ (resp. $\operatorname{Var}(X))$. The covariance of two random variables $X$ and $Y$ is noted $\operatorname{Cov}(X, Y)$. Let $\bar{X}$ be the empirical mean $1 / T \sum_{t=1}^{T} X(t)$ of any independent random process $(X(t))_{1 \leq t \leq T}$. Let us recall that if $X$ has 0 mean, then $\overline{X^{2}}$ is an asymptotically unbiased estimator of the variance of $X$. Using (2) and (3),

$$
\begin{aligned}
\bar{v}(x, y)=s(x, y)+(\bar{\alpha}, \bar{\beta}) \nabla s(x, y)+\frac{1}{2} \overline{\alpha^{2}} \frac{\partial^{2} s}{\partial x^{2}}(x, y)+\frac{1}{2} \overline{\beta^{2}} \frac{\partial^{2} s}{\partial y^{2}}(x, y)+ & \overline{\alpha \beta} \frac{\partial^{2} s}{\partial x \partial y}(x, y) \\
& +\frac{1}{T} \sum_{t} n_{s\left(x+\alpha_{t}, y+\beta_{t}\right)}(x, y, t)
\end{aligned}
$$

Since the expectation of the noise $n$ is zero, and $\alpha_{t}, \beta_{t}$ are also 0 -mean random variables (yielding $E\left(\overline{\alpha^{2}}\right)=\operatorname{Var}(\alpha), E\left(\overline{\beta^{2}}\right)=\operatorname{Var}(\beta)$, and $\left.E(\overline{\alpha \beta})=\operatorname{Covar}(\alpha, \beta)\right)$, this proves the following proposition. 
Proposition 2.1 The expectation of the averaged image $\bar{v}$ is

$$
E(\bar{v})=s+\frac{1}{2} \operatorname{Var}(\alpha) \frac{\partial^{2} s}{\partial x^{2}}+\frac{1}{2} \operatorname{Var}(\beta) \frac{\partial^{2} s}{\partial y^{2}}+\operatorname{Covar}(\alpha, \beta) \frac{\partial^{2} s}{\partial x \partial y}
$$

or in a compact manner:

$$
E(\bar{v})=s+\frac{1}{2} \operatorname{div}(\operatorname{Var}(\alpha, \beta) \cdot \nabla s)
$$

where $\operatorname{div}(F)$ is the divergence of any vector field $F$ and $\operatorname{Var}(\alpha, \beta)$ is the variance-covariance matrix of the $2 D$ process $\left(\alpha_{t}, \beta_{t}\right)$.

Within this model, the averaged intensity at $(x, y)$ may be a biased estimator of $s(x, y)$, depending on the second derivatives values with respect to the variance-covariance matrix. In the following, the pattern pitch of a typical grid image $s$ being limited to a few pixels, the second derivatives are likely to be relatively large at every pixel, contrary to natural images where large iso-intensity regions are likely to appear. This remark also holds for random patterns used in digital image correlation for instance [26]. An averaged grid image $\bar{v}$ is thus likely to be a biased estimator of the ideal, vibration- and noise-free image $s$.

Let us carry on with the calculation of $\bar{v}$ by specializing to grid images and by using some information about the physical quantities which are involved. The reference image $s$ satisfies (1). Its first and second derivatives are given by the chain rule. For example,

$$
\frac{\partial s}{\partial x}(x, y)=\frac{\gamma A}{2}\left(2 \pi f+\frac{\partial \phi_{1}}{\partial x}(x, y)\right) \ell^{\prime}\left(2 \pi f x+\phi_{1}(x, y)\right)+\frac{\gamma A}{2} \frac{\partial \phi_{2}}{\partial x}(x, y) \ell^{\prime}\left(2 \pi f y+\phi_{2}(x, y)\right)
$$

It has been reminded in the introduction that the problem of interest is characterized by the tiny values of the derivatives of $\phi$ compared to $2 \pi f$. It is thus legitimate to simplify (7) into

$$
\frac{\partial s}{\partial x}(x, y)=\frac{\gamma A}{2} 2 \pi f \ell^{\prime}\left(2 \pi f x+\phi_{1}(x, y)\right)
$$

Similarly, the first and second derivatives of $s$ simplify into

$$
\begin{aligned}
\frac{\partial s}{\partial y}(x, y) & =\frac{\gamma A}{2} 2 \pi f \ell^{\prime}\left(2 \pi f y+\phi_{2}(x, y)\right) \\
\frac{\partial^{2} s}{\partial x^{2}}(x, y) & =\frac{\gamma A}{2}(2 \pi f)^{2} \ell^{\prime \prime}\left(2 \pi f x+\phi_{1}(x, y)\right) \\
\frac{\partial^{2} s}{\partial y^{2}}(x, y) & =\frac{\gamma A}{2}(2 \pi f)^{2} \ell^{\prime \prime}\left(2 \pi f y+\phi_{2}(x, y)\right) \\
\frac{\partial^{2} s}{\partial x \partial y}(x, y) & =0
\end{aligned}
$$

where $\ell^{\prime}$ and $\ell^{\prime \prime}$ are respectively the first and second derivatives of the 1-D $2 \pi$-periodic function $\ell$.

If the Fourier series expansion of $\ell$ writes $\sum_{k \in \mathbb{Z}} d_{k} e^{i k}$, then the expansion of $\ell^{\prime}$ writes $\sum_{k \in \mathbb{Z}} i k d_{k} e^{i k}$ and the expansion of $\ell^{\prime \prime}$ writes $-\sum_{k \in \mathbb{Z}} k^{2} d_{k} e^{i k}$.

With (4) and the simplifications given by (8-12), the averaged grid image $\bar{v}$ thus satisfies the following proposition.

Proposition 2.2 An averaged grid image $\bar{v}$ is such that:

$$
\bar{v}(x, y)=\frac{A}{2}\left(2+\gamma \ell_{1}\left(2 \pi f x+\phi_{1}(x, y)\right)+\gamma \ell_{2}\left(2 \pi f y+\phi_{2}(x, y)\right)\right)+\frac{1}{T} \sum_{t} n_{s\left(x+\alpha_{t}, y+\beta_{t}\right)}(x, y, t)
$$


where the Fourier series expansion of $\ell_{1}$ writes

$$
\begin{aligned}
\ell_{1}(x) & =\sum_{k \in \mathbb{Z}}\left(1+i 2 \pi f k \bar{\alpha}-\frac{1}{2}(2 \pi f)^{2} k^{2} \overline{\alpha^{2}}\right) d_{k} e^{i k x} \\
& =l(x)+\sum_{k \in \mathbb{Z}}\left(i 2 \pi f k \bar{\alpha}-\frac{1}{2}(2 \pi f)^{2} k^{2} \overline{\alpha^{2}}\right) d_{k} e^{i k x}
\end{aligned}
$$

and the same relation holds for $\ell_{2}$ with $\beta$ instead of $\alpha$, namely:

$$
\begin{aligned}
\ell_{2}(x) & =\sum_{k \in \mathbb{Z}}\left(1+i 2 \pi f k \bar{\beta}-\frac{1}{2}(2 \pi f)^{2} k^{2} \overline{\beta^{2}}\right) d_{k} e^{i k x} \\
& =l(x)+\sum_{k \in \mathbb{Z}}\left(i 2 \pi f k \bar{\beta}-\frac{1}{2}(2 \pi f)^{2} k^{2} \overline{\beta^{2}}\right) d_{k} e^{i k x}
\end{aligned}
$$

When the number of images is large enough to identify $\bar{\alpha}$ with $E(\alpha)(=0)$ and $\overline{\alpha^{2}}$ with $\operatorname{Var}(\alpha)$ (resp. $\bar{\beta}$ with $E(\beta)$ and $\overline{\beta^{2}}$ with $\left.\operatorname{Var}(\beta)\right)$, then $\ell_{1}(x)$ simplifies into $\ell_{1}(x)=\ell(x)-\sum_{k \in \mathbb{Z}} \frac{1}{2}(2 \pi f)^{2} k^{2} \operatorname{Var}(\alpha) d_{k} e^{i k x}$ (resp. $\left.\ell_{2}(x)=\ell(x)-\sum_{k \in \mathbb{Z}} \frac{1}{2}(2 \pi f)^{2} k^{2} \operatorname{Var}(\beta) d_{k} e^{i k x}\right)$. In accordance with the intuition, one can see that vibrations perfectly distributed along the $x$ - (resp. $y$-) axis affect the grid line perpendicular to this direction, and that the effect is all the larger as the vibration amplitude (proportional to $\operatorname{Std}(\alpha)$ ) is large with respect to the pattern pitch $(p=1 / f)$.

By definition of $n$, the expectation of the noise component in (13) is zero. A straightforward computation gives the following property on the variance.

Proposition 2.3 The variance of the averaged grid image is such that:

$$
\operatorname{Var}\left(\frac{1}{T} \sum_{t} n_{s\left(x+\alpha_{t}, y+\beta_{t}\right)}(x, y, t)\right)=\mathcal{O}(1 / T)
$$

Indeed, in the Poisson-Gaussian noise model commonly adopted for camera sensor noise [9], a straightforward calculation shows that the noise variance linearly depends on the expected intensity $[7,8,12,22]$. Noting $K$ and $L$ respectively the slope and the intercept of this linear relation, hence $K l+L$ the variance of the noise $n_{l}$ affecting a pixel of expected intensity $l$, the variance of the noise component in (13) satisfies

$$
\begin{aligned}
& \operatorname{Var}\left(\frac{1}{T} \sum_{t} n_{s\left(x+\alpha_{t}, y+\beta_{t}\right)}(x, y, t)\right)=\frac{1}{T^{2}} \sum_{t}\left(K s\left(x+\alpha_{t}, y+\beta_{t}\right)+L\right) \\
&=\frac{K}{T}(s(x, y)+(\bar{\alpha}, \bar{\beta}) \nabla s(x, y) \\
&\left.+\frac{1}{2} \bar{\alpha}^{2} \frac{\partial^{2} s}{\partial x^{2}}(x, y)+\frac{1}{2} \overline{\beta^{2}} \frac{\partial^{2} s}{\partial y^{2}}(x, y)+\overline{\alpha \beta} \frac{\partial^{2} s}{\partial x \partial y}(x, y)\right)+\frac{L}{T^{2}} \\
&=\mathcal{O}(1 / T)
\end{aligned}
$$

To sum up, we can say that the average grid image is a biased estimation of the unknown noise-free grid (cf Prop. 2.1), and that, according to Prop. 2.2, it is equivalent to a grid image whose line profiles along each direction has been modified into $\ell_{1}$ and $\ell_{2}$, added to a $\mathcal{O}(1 / T)$ noise component (Prop. 2.3). The next section quantifies to what extent the phase and phase derivative maps (hence displacements and strain components) are affected by the bias induced by the vibrations in the averaged grid image. 


\subsection{Estimating the phase and phase derivative from the averaged grid image}

The classic route to estimate the phase maps $\phi_{1}$ and $\phi_{2}$ (and their derivatives) from any grid image $u$, either averaged or not, is to use the windowed Fourier transform $[4,25]$. Let us note

$$
\begin{aligned}
& \Psi_{1}(v)(x, y)=\int_{\mathbb{R}^{2}} v(\xi, \eta) g_{\sigma}(\xi-x, \eta-y) e^{-2 i \pi f \xi} \mathrm{d} \xi \mathrm{d} \eta \\
& \Psi_{2}(v)(x, y)=\int_{\mathbb{R}^{2}} v(\xi, \eta) g_{\sigma}(\xi-x, \eta-y) e^{-2 i \pi f \eta} \mathrm{d} \xi \mathrm{d} \eta
\end{aligned}
$$

In practice $g_{\sigma}$ is a Gaussian window of standard deviation $\sigma$. The calculated $\Psi_{1}$ and $\Psi_{2}$ are used in [4] (after [25]) to estimate $\phi_{1}$ and $\phi_{2}$. Since the phase derivatives are very small with respect to $2 \pi f$, it is proved in $[20,23]$ that the following approximations are actually valid:

$$
\begin{array}{r}
\arg \left(\Psi_{1}(v)\right)=\arg \left(d_{1}\right)+g_{\sigma} * \phi_{1}+n \\
\frac{\partial \arg \left(\Psi_{1}(v)\right)}{\partial \cdot}=g_{\sigma} * \frac{\partial \phi_{1}}{\partial \cdot}+n^{\prime}
\end{array}
$$

where $*$ denotes the $2 \mathrm{D}$ convolution, and $d_{1}$ denotes as above the first Fourier coefficient of the periodic line pattern $\ell$, and $n$ and $n^{\prime}$ are spatially correlated 0 -mean noise processes. (The same equations as (24)-(25) hold for $\Psi_{2}$ and $\phi_{2}$.)

In the case of a homoscedastic noise, it is proved in [23] that the variances of $n$ and $n^{\prime}$ are proportional to $v /\left(\sigma^{2}\left|d_{1}\right|^{2}\right)$ and $v /\left(\sigma^{4}\left|d_{1}\right|^{2}\right)$ respectively, where $v$ is the variance of the noise in the grid image and $|\cdot|$ is the norm of any complex number. In the case of a realistic signal-dependent Poisson-Gaussian noise, it is shown in [10] that this characterization is still valid provided the generalized Anscombe transform [18] has been applied to the images to stabilize the variance beforehand. A typical example of a realistic standard deviation of the noise in the phase maps is $3 \cdot 10^{-3} \mathrm{rad}$ and $5 \cdot 10^{-4} \mathrm{rad} \cdot \mathrm{pixel}^{-1}$ in the phase derivative maps [10].

It is important to note that (24-25) and the remark above mean that a compromise must be found between a large $\sigma$ which smooths out the noise in the phase and phase derivative and a small $\sigma$ which makes $g_{\sigma} * \phi_{1}$ to get closer to the sought phase $\phi_{1}$. Consequently, reducing the noise in the grid image (before phase estimation) would make it possible to use smaller $\sigma$, up to the limit reminded in [23] which imposes $\sigma \geq 1 / f$. We can see that the vibrations change the $\left|d_{1}\right|$ term into $\left|1-\frac{1}{2}(2 \pi f)^{2} \operatorname{Var}(\alpha)\right|\left|d_{1}\right|$ obtained by simplifying (14) for large $T$, which is smaller than $\left|d_{1}\right|$. In spite of this negative impact on the variance of $n$ and $n^{\prime}$, time-averaging is still valuable because of the $\mathcal{O}(1 / T)$ decreasing of the noise in the grid image (Prop. 2.3).

In the remainder of this section, we quantify the convergence rate of the phase and phase derivative estimated from the averaged grid to the unknown phase and phase derivative from the noise-free grid. From (24)-(25) and the averaged grid $\bar{v}$ given by (13):

$$
\begin{aligned}
& \arg \left(\Psi_{1}(\bar{v})\right)(x, y)=\arg \left(\Psi_{1}(s)\right)(x, y)+\delta_{1}+n_{T}(x, y) \\
& \frac{\partial \arg \left(\Psi_{1}(\bar{v})\right)}{\partial \cdot}(x, y)=\frac{\partial \arg \left(\Psi_{1}(s)\right)}{\partial \cdot}(x, y)+n_{T}^{\prime}(x, y)
\end{aligned}
$$

where as above $s$ is the reference grid, $n_{T}$ and $n_{T}^{\prime}$ have a variance proportional to the variance of the noise in $\bar{v}$ (hence decreasing in $1 / T$ from $(21)$ ), and

$$
\delta_{1}=\arg \left(\left(1+2 i \pi f \bar{\alpha}-\frac{1}{2}(2 \pi f)^{2} \overline{\alpha^{2}}\right) d_{1}\right)-\arg \left(d_{1}\right)
$$


A simple calculation (see appendix) proves that:

$$
\delta_{1}=\arctan \left(\frac{2 \pi f \bar{\alpha}}{1-\frac{1}{2}(2 \pi f)^{2} \overline{\alpha^{2}}}\right)
$$

The same relation holds for $\delta_{2}$ with respect to $\beta$ :

$$
\delta_{2}=\arctan \left(\frac{2 \pi f \bar{\beta}}{1-\frac{1}{2}(2 \pi f)^{2} \overline{\beta^{2}}}\right)
$$

It can be observed that $\delta_{1}$ and $\delta_{2}$ do not depend on $d_{1}$.

The question is now, how does the additional $\delta_{1}$ (resp. $\left.\delta_{2}\right)$ term affect the estimation of $\arg \left(\Psi_{1}(s)\right)$ $\left(\right.$ resp. $\arg \left(\Psi_{2}(s)\right)$ ? Under the mild assumption that $\bar{\alpha}$ follows a symmetric law, and considering $\overline{\alpha^{2}}$ equal to $\operatorname{Var}(\alpha)$, then the expectation of $\delta_{1}$ is 0 . One has indeed $E(h(X))=0$ as soon as $h(=\arctan )$ is an odd function and $X\left(=2 \pi f \bar{\alpha} /\left(1-(2 \pi f)^{2} \operatorname{Var}(\alpha) / 2\right)\right)$ has a symmetric distribution function. Estimating the variance of $\delta_{1}$ is a bit tricky but an approximation is given by the delta-method [19]: $\operatorname{Var}(h(X)) \simeq\left(h^{\prime}(E(X))\right)^{2} \operatorname{Var}(X)$. Here, $E(X)=0$ hence $h^{\prime}(E(X))=1$. The variance of $\delta_{1}$ is thus given by the following proposition.

Proposition 2.4 Under the above-mentioned assumptions, $\delta_{1}$ is a 0 -mean random variable whose variance is given by:

$$
\operatorname{Var}\left(\delta_{1}\right) \simeq\left(\frac{2 \pi f}{1-\frac{1}{2}(2 \pi f)^{2} \operatorname{Var}(\alpha)}\right)^{2} \operatorname{Var}(\bar{\alpha})
$$

A similar formula holds for $\delta_{2}$.

An experimental assessment (cf [22]) shows that in a typical case the peak-to-peak amplitude of the vibrations can be up to $10 \%$ of the size of a pixel on the specimen. The standard deviation of $\alpha$ is hence around 0.05 (twice the standard deviation being approximately equal to 0.1 ). With $f$ being typically equal to $1 / 5$ pixel $^{-1}$, the variance of $\delta_{1}$ is approximately $(1.34)^{2} \operatorname{Var}(\bar{\alpha})$. Now, the variance of the sample mean $\bar{\alpha}$ is known to be $2 \operatorname{Var}^{2}(\alpha) /(T-1)$ if $\alpha$ is a Gaussian process [14]. The resulting standard deviation of $\delta_{1}$ is therefore approximately $1.34 \times \sqrt{2} \times 0.05 / \sqrt{T-1} \simeq 0.1 / \sqrt{T}$.

The estimated phase thus differs from the actual phase in a 0 -mean spatially correlated noise $n_{T}$ whose standard deviation decreases as $1 / \sqrt{T}$, added to a spatially constant 0 -mean random $\delta$ whose standard deviation is proportional to $\operatorname{Std}(\bar{\alpha})=\mathcal{O}(1 / \sqrt{T})$, cf. $(26,29,31)$. The error $\delta$ is likely to attain values as large as $2 \operatorname{Std}\left(\delta_{1}\right)$, which may be larger than the standard deviation of the noise term in the case of strong vibrations, as illustrated in Section 3. Concerning the estimated phase derivatives, they turn out to be independent from the vibrations $\left(\alpha_{t}, \beta_{t}\right)$. It only differs from the actual value in a 0 -mean correlated noise $n_{T}^{\prime}$ decreasing as $1 / \sqrt{T}$, cf. (27).

The method for retrieving the phases and their derivatives from biased averaged grid, which is described in this section, is called method $\mathbf{A}$.

\subsection{Averaging phases and phase derivatives estimated from each of the grid images separately}

Instead of estimating the phase from the biased averaged grid, it is possible to estimate the phase from each of the $T$ images separately with (24-25), and to reduce noise afterwards by averaging the $T$ phase maps. 
With the same notation as above, the averaged phase and phase derivatives satisfy (the same equations hold for $\Psi_{2}$ ):

$$
\begin{aligned}
& \overline{\arg \left(\Psi_{1}(v)\right)}=\overline{\delta_{1}}+\arg \left(\Psi_{1}(s)\right)+\bar{n} \\
& \frac{\overline{\partial \arg \left(\Psi_{1}(v)\right)}}{\partial \cdot}=\frac{\partial \arg \left(\Psi_{1}(s)\right)}{\partial \cdot}+\overline{n^{\prime}}
\end{aligned}
$$

where the averaged noises $\bar{n}$ and $\overline{n^{\prime}}$ decrease as $1 / \sqrt{T}$, and $\overline{\delta_{1}}$ is the average of the $\arctan \left(2 \pi f \alpha_{t} /\left(1-\left(2 \pi f \alpha_{t}\right)^{2} / 2\right)\right)$ whose expectation is 0 as soon as $\alpha_{t}$ has a symmetric distribution function. The estimation of the variance of $\overline{\delta_{1}}$ obtained by the delta-method is: $\operatorname{Var}\left(\delta_{1}\right) \simeq(2 \pi f)^{2} \operatorname{Var}(\alpha)$, yielding a $\mathcal{O}(1 / T)$ decreasing of the variance of $\delta_{1}$.

This results in an alternative method to smooth out the noise in the phase and phase derivative maps, which is much more computationally intensive since it necessitates to compute $T$ windowed Fourier transform (for each of the phases or phase derivatives), instead of a single transform (over the averaged grid image) with method $\mathbf{A}$.

The method described in this section is called method $\mathbf{B}$.

\section{Experiments}

Methods A (Section 2.2) and $\mathbf{B}$ (Section 2.3) are based on approximations. It is necessary to experimentally assess their validity. Following the discussion in Section 2.2, the value of the size $\sigma$ of the analysis window is chosen to be equal to the pattern pitch $p$ which is the minimum admissible value [23]. In Sections 3.1 and $3.2, \sigma=p=5$ pixels.

\subsection{Real data set}

This first example illustrates the effect of time averaging on a set of real strain maps. A tensile test was performed on an open-hole specimen made of aluminum (thickness: $2 \mathrm{~mm}$, width: $50 \mathrm{~mm}$, length: $250 \mathrm{~mm}$, diameter of the hole: $12 \mathrm{~mm}$ ). A heterogeneous strain field is induced around the hole. Hundred images were taken with a Sensicam QE camera in the reference configuration as well as for a force applied equal to $5000 \mathrm{~N}$. The two series are impaired by mechanical vibrations. Of course, the vibrations do not equally affect the two series.

Once $\phi_{1}$ and $\phi_{2}$ are estimated from a grid image before and after deformation as explained above, it is possible to derive the in-plane displacement $u_{x}$ and $u_{y}$ in the $x$ - and $y$-directions by forming the following phase variations:

$$
\left\{\begin{array}{l}
u_{x}=-\frac{p}{2 \pi} \Delta \phi_{1} \\
u_{y}=-\frac{p}{2 \pi} \Delta \phi_{2}
\end{array}\right.
$$

where $p=1 / f$ is the pattern pitch ( $\operatorname{cf}(1))$ and $\Delta \phi_{i}$ denotes the difference of the phases before and after deformation. The linearized strain components are eventually given by the symmetrized part of the displacement gradient [2]. Thus:

$$
\left\{\begin{array}{l}
\varepsilon_{x x}=\frac{\partial u_{x}}{\partial x}=-\frac{p}{2 \pi} \Delta \frac{\partial \phi_{1}}{\partial x} \\
\varepsilon_{y y}=\frac{\partial u_{y}}{\partial y}=-\frac{p}{2 \pi} \Delta \frac{\partial \phi_{2}}{\partial y} \\
2 \varepsilon_{x y}=\frac{\partial u_{x}}{\partial y}+\frac{\partial u_{y}}{\partial x}=-\frac{p}{2 \pi}\left(\Delta \frac{\partial \phi_{1}}{\partial y}+\Delta \frac{\partial \phi_{2}}{\partial x}\right)
\end{array}\right.
$$

Figure 2 shows the time evolution of some values of the displacement $u_{x}$ in the $x$-direction (resp. of the displacement $u_{y}$ in the $y$-direction) estimated from the $t$-th images of each series. While the average 

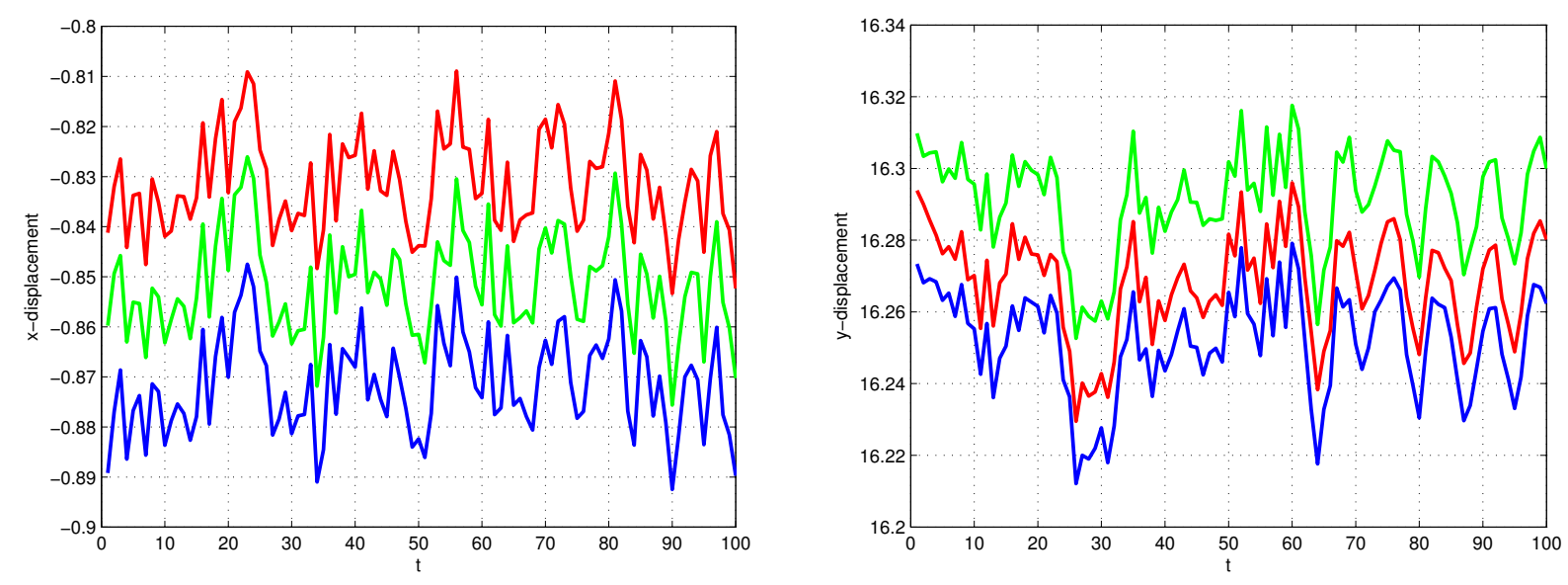

Figure 2: Three plots of $u_{x}(x, y)$ (left) and $u_{y}(x, y)$ (right) measured at three distant locations on the grid. In-phase fluctuations are caused by the residual vibrations.

displacement depends on the local deformation of the specimen (added to a noise component), in-phase fluctuations correspond to the residual vibrations in the $x$ - (resp. $y$-) direction. A translation of the reference grid of $\alpha_{t}$ along the $x$-direction gives an additional $2 \pi f \alpha_{t}$ displacement. The standard deviation of this additional displacement is hence $2 \pi f \operatorname{Std}(\alpha)$. The measured displacement being obtained by $(34)$, the standard deviation on $u_{x}$ is $\sqrt{2} 2 \pi f \operatorname{Std}(\alpha) \frac{p}{2 \pi}=\sqrt{2} \operatorname{Std}(\alpha)$ (the result is multiplied by $\sqrt{2}$ to account for the fact that two phase maps are subtracted to calculate a displacement map). In this experiment, we have measured a standard deviation equal to 0.01 in the $x$-direction and to 0.015 in the $y$-direction, hence $\operatorname{Std}(\alpha) \simeq 0.007$ and $\operatorname{Std}(\beta) \simeq 0.01$.

Figure 3 shows the benefit of time averaging on the strain map in terms of visual aspect since the noise due to the sensor noise propagation through the image processing procedure progressively decreases. One can see that the noise component (the elongated "blobs" clearly visible in the $T=1$ case) vanishes in the strain maps when the number of averaged grid images grows, yielding a clearer visualization of the phenomena of interest. Some straight shapes can be noticed in the $T=100$ case. They are not likely to have a mechanical explanation and are certainly caused by manufacturing defects of the grid.

It must be pointed out that no ground truth is really available here: a closed-form solution for the open-hole specimen is available within the framework of elasticity [28], but boundary conditions actually applied during the test may differ from those applied in the model. The same remark holds for the material properties. It is therefore not really possible to check here that the averaged strain map is affected or not by a bias due to the fact that the averaged grid image is a biased estimator of the noise-free grid image. The simulations presented in the section below were carried out for this purpose. 

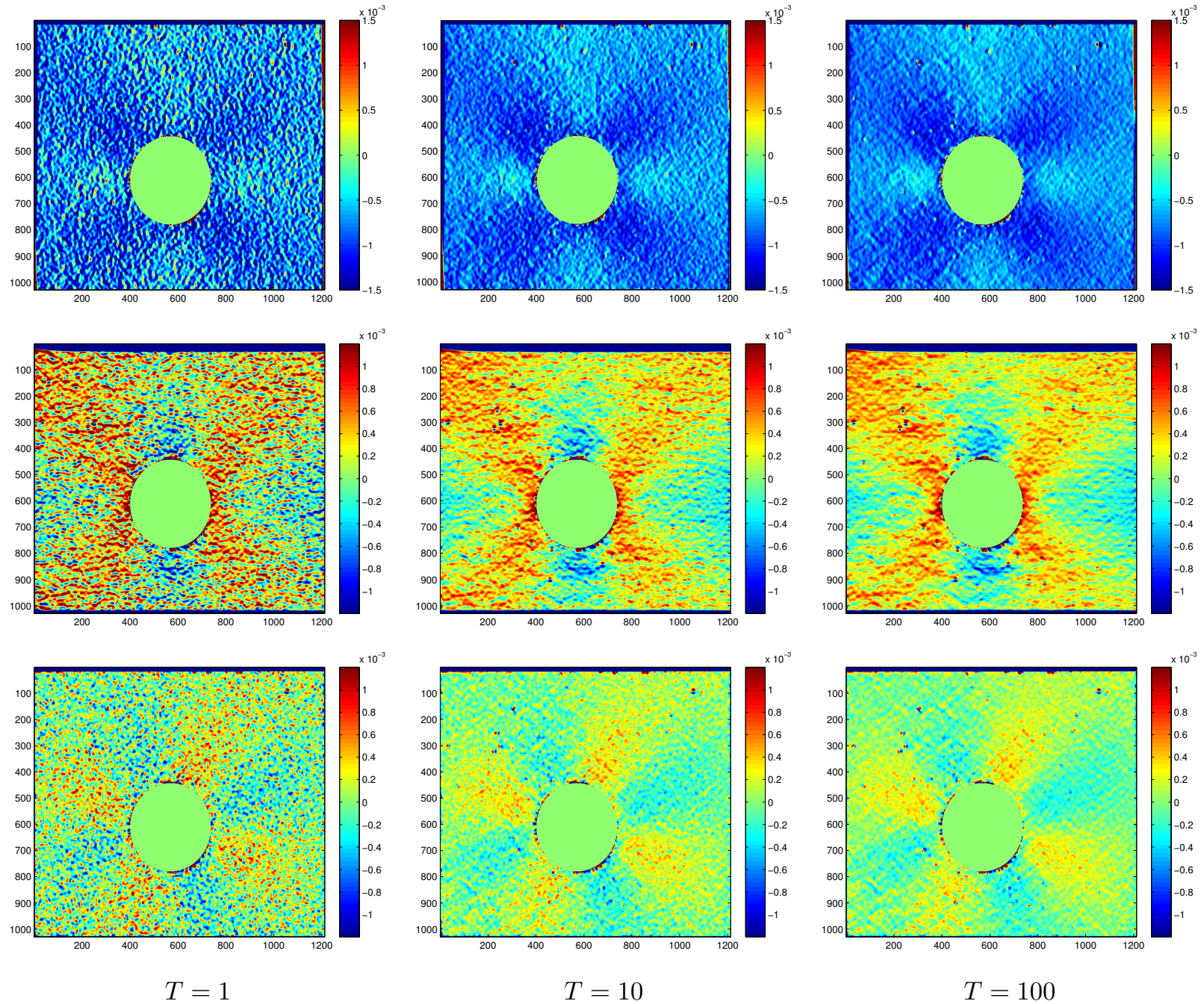

Figure 3: Evolution of the strain components $\varepsilon_{x}$ (top), $\varepsilon_{y}$ (middle), $\varepsilon_{x y}$ (bottom) estimated on the averaged grid obtained from $T=\{1,10,100\}$ images. ( $T=1$ corresponds to a raw image, without any averaging.) The $x$ - and $y$ - axis are respectively the vertical and horizontal axis. Each pixel represents a surface whose area is $40 \times 40$ micrometers $^{2}$ on the specimen. 


\subsection{Synthetic data set}

Synthetic grid images are generated following (1)-(2), with $A=2^{12}, \gamma=0.9, f=1 / 5$, and $l(x)=\sin ^{3}(x)$. These values are realistic compared to the specimen of interest. Such an $l$ function is chosen to simulate sharp lines. The phase maps $\phi_{1}$ and $\phi_{2}$ are here synthetic (discrete) images depicted in Figure 4, normalized such that the maximum of phase derivatives is equal to 0.005 , which is a realistic value for strain components observed in structural materials under load. This gives a maximum value equal to 0.06 for $\phi_{1}$ and to 0.42 for $\phi_{2}$. The image $s\left(x+\alpha_{t}, y+\beta_{t}\right)$ impaired by a vibration $\left(\alpha_{t}, \beta_{t}\right)$ is built by bilinearly interpolating the phase maps. Four experiments are led, based on four representative parameters for the law of $\left(\alpha_{t}, \beta_{t}\right)$. In each case, $\alpha_{t}$ and $\beta_{t}$ follow a Gaussian distribution, with respective standard deviations (expressed in pixels): $\left(\sigma_{\alpha}, \sigma_{\beta}\right)=(0.15,0.1)$ (case 1$),\left(\sigma_{\alpha}, \sigma_{\beta}\right)=(0.1,0.05)$ (case 2$),\left(\sigma_{\alpha}, \sigma_{\beta}\right)=$ $(0.01,0.01)$ (case 3$),\left(\sigma_{\alpha}, \sigma_{\beta}\right)=(0.001,0.005)$ (case 4$)$. Case 1 corresponds to strong vibrations which may be reached if no care is paid to this aspect in the experimental setting. Case 3 is representative of the typical residual vibrations measured in the experiment of Section 3.1. A time- and spatially independent heteroscedastic noise with the same parameters as the Sensicam QE camera is added [22] and the synthetic grid image is quantized over 12 bits. Consequently, the phase derivatives (whose maximum amplitude is 0.005) hardly exceed the noise floor reminded in Section 2.2. The resulting image follows the model of (2) (apart from quantization). Note that some phase information is irremediably lost by quantization.

To simplify the presentation, we focus here on the phase and phase derivative estimation, though the mechanical quantities of interest are the displacement and strain maps calculated through (34-35).

Figure 5 a shows a close-up on the averaged grid ( $T=100$ images are used), in the representative case 2 . The difference between the averaged grid and the ideal reference grid (that is, without any noise and vibration) is shown in Figure $5 \mathrm{~b}$. It depicts the bias affecting the sample mean. The averaged grid image does indeed not converge to the vibration- and noise-free grid image $s$, as predicted by (13). The RMSE, defined as the root-mean-square error between the averaged and reference grids, hence does not converge to 0 as the number of averaged images increases, cf. Figure $5 \mathrm{c}$.

Figures 6 to 11 show the phase and phase derivative maps obtained from the average of $T$ grid images, with $T=\{1,10,200\}$ (method $\mathbf{A}$, since method $\mathbf{B}$ gives visually similar results). The ground truth (noted GT) is also given; it corresponds here to the phases and phase derivatives obtained from the noise-free synthetic grid image. We can see that the correlated noise described in $[11,23]$ gives small "blobs" which are actually smoothed out when $T$ increases, the maps visually converging to the ground truth. Note that the aspect of the noise is visually similar to what is observed in the real strain maps of Section 3.1.

A quantitative assessment can be obtained through a ground truth. The ground truth is given here by the phase and phase derivative maps obtained from $s(x, y))$ by the classic windowed Fourier transform approach, without any added noise and vibrations. The RMSE of a quantity estimated with the averaged grid is defined as the root-mean-square error between this estimate and the corresponding ground truth. It is an estimation of the standard deviation error which should be compared to 0.001 for the phase derivatives, to 0.012 for $\phi_{1}$, and to 0.085 for $\phi_{2}$. The RMSE is computed for a varying number $T$ of averaged images. The larger the amplitude of the vibrations, the larger the RMSE.

Figure 12 shows the RMSE plots for method A. Up to $T=1,000$ images are considered to emphasize the $1 / T$ behaviour. As predicted by $(27)$, the RMSE of the phase derivatives does not depend on $\left(\sigma_{\alpha}, \sigma_{\beta}\right)$ (the curves in graphs e, $\mathrm{f}, \mathrm{g}, \mathrm{h}$ are superposed), and decreases in $1 / \sqrt{T}$. For example, with $T=200$ images, the RMSE is smaller than $3 \cdot 10^{-5}$ radian.pixel $^{-1}$. The RMSE of the phases (graphs a and b) seem to decrease but now depend on $\left(\sigma_{\alpha}, \sigma_{\beta}\right)$ in accordance with (26), (29) and (30). The strong vibrations of cases 1 and 2 especially affect the RMSE plots. However, with $T=200$, the RMSE is still below 0.03 radian for $\phi_{1}$ (resp. 0.01 for $\phi_{2}$ ) in any case, and is smaller than 0.002 (resp. 0.001 ) in cases 3 
and 4 . This means that cases 1 and 2 strongly affect the phase maps $\phi_{1}$ and $\phi_{2}$ (whose maximum values are respectively 0.012 and 0.085 ). Subtracting the value of $\delta$. given by (29) (sample means and variance of $\left(\alpha_{t}, \beta_{t}\right)$ are known in this synthetic data set) to the phase retrieved on the averaged grid gives the so-called "corrected phase". The RMSE of the corrected phases (c and d) are much smaller, decrease in $1 / \sqrt{T}$, and do not depend anymore on $\left(\sigma_{\alpha}, \sigma_{\beta}\right)$ for cases 3 and 4 . A residual error can still be seen in case 1 and for $\phi_{1}$ in case 2 , which corresponds to a standard deviation of the vibrations larger than 0.1.

Method $\mathbf{B}$ gives plots which are comparable to method $\mathbf{A}$, as illustrated in Figure 13. However, the computation time of method $\mathbf{A}$ is $8 \mathrm{sec}$. for a stack of 100 images, while it is 118 sec. for $\mathbf{B}$ (Matlab code, Intel Xeon E3-1240 processor). The same results were observed for the real data set of Section 3.1.

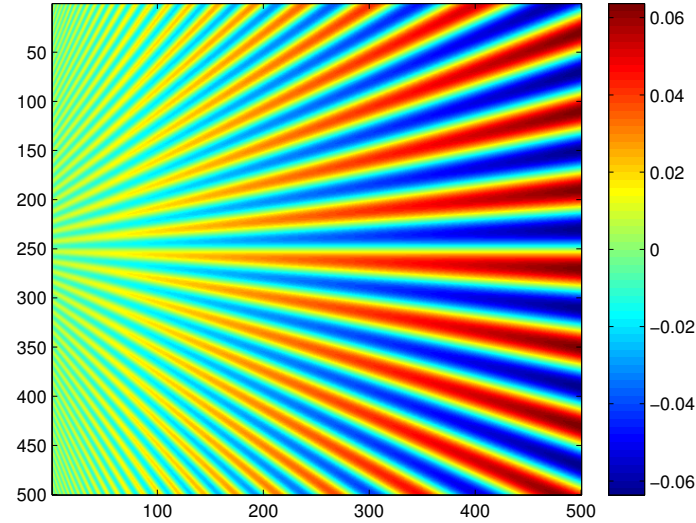

a.

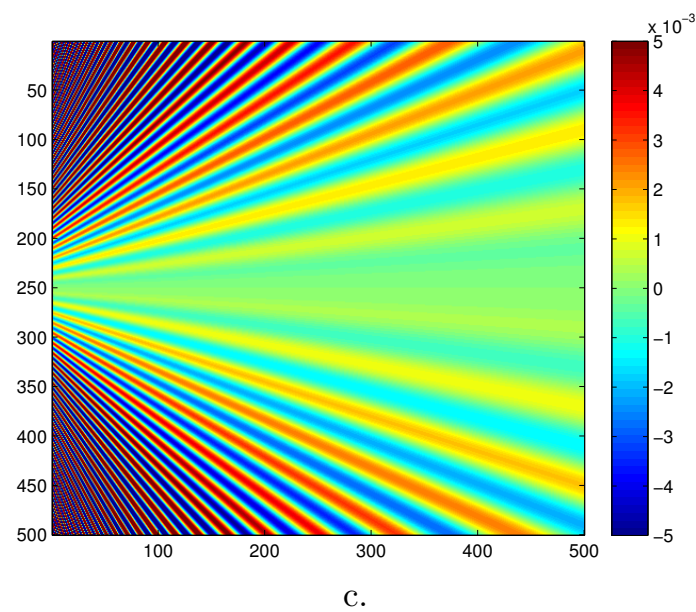

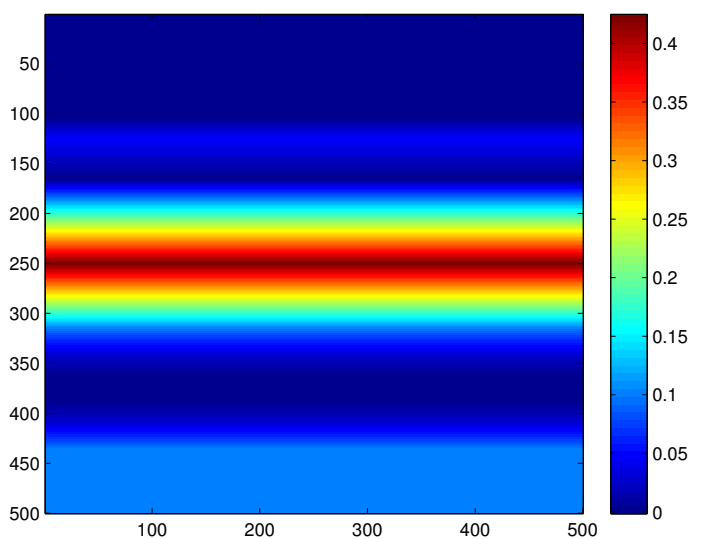

b.

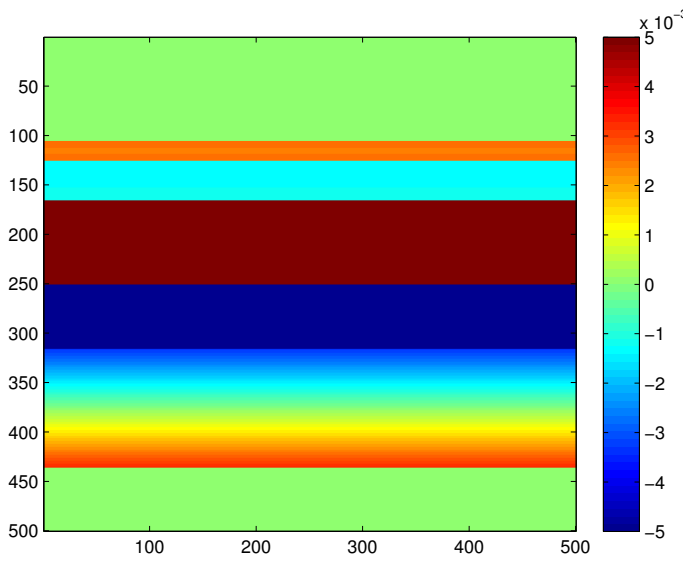

d.

Figure 4: a: Synthetic $\phi_{1}$. b: Synthetic $\phi_{2}$. c: corresponding $\partial \phi_{1} / \partial x$. d: corresponding $\partial \phi_{2} / \partial y$. The phase $\phi_{1}$ is built as a sine wave along the vertical axis whose frequency continuously varies along the horizontal axis, and the phase $\phi_{2}$ as a piecewise quadratic function (hence $\partial \phi_{2} / \partial y$ is non-continuous piecewise linear). Such functions are chosen to mimic realistic phase or phase derivative maps, with abrupt changes or high-frequency phenomena. 


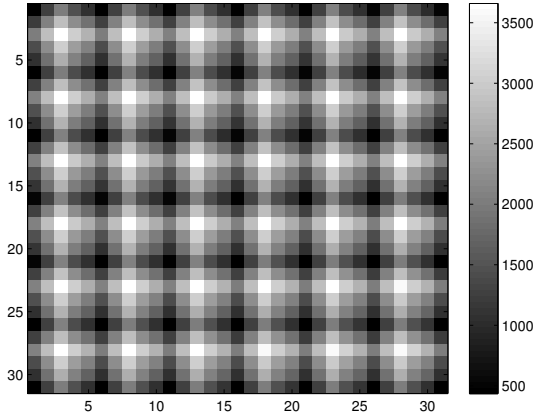

a.

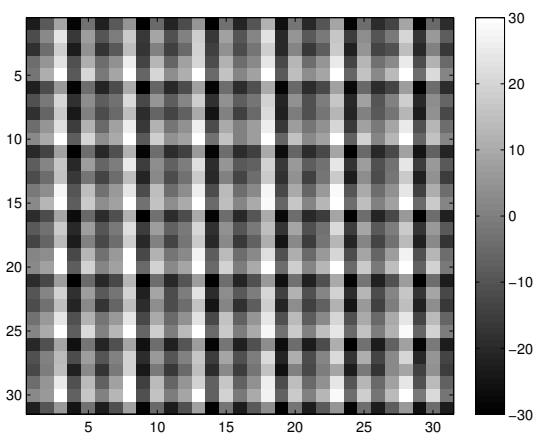

b.

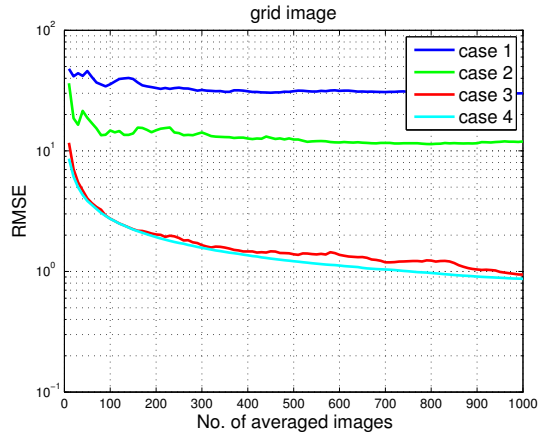

c.

Figure 5: a: A close-up on the averaged grid. b: The same close-up on the difference between the averaged grid and the reference grid. c: RMSE for the averaged grid (with $T$ up to 1,000; $\log$ scale).
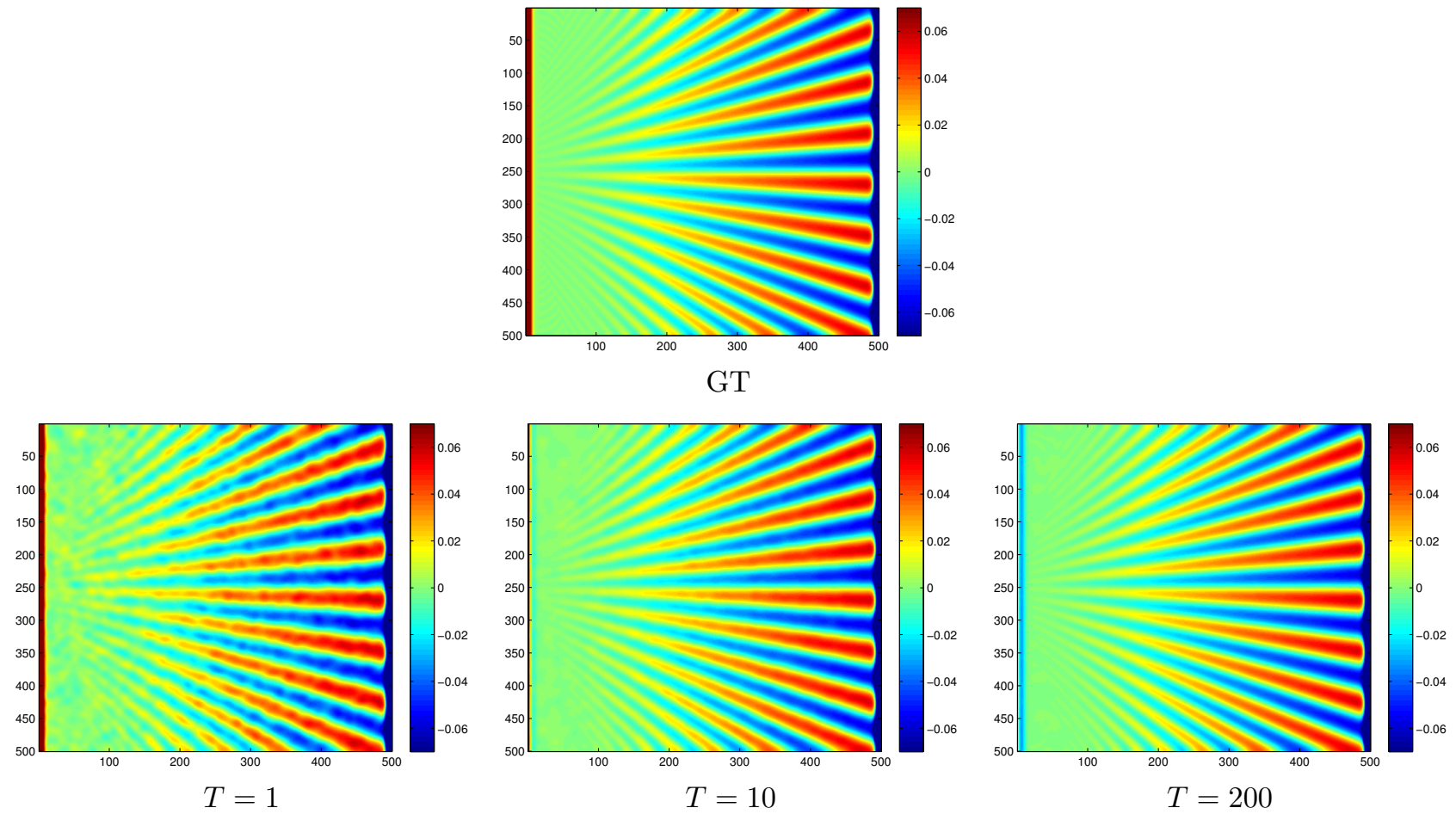

Figure 6: Method A. $\phi_{1}$ 

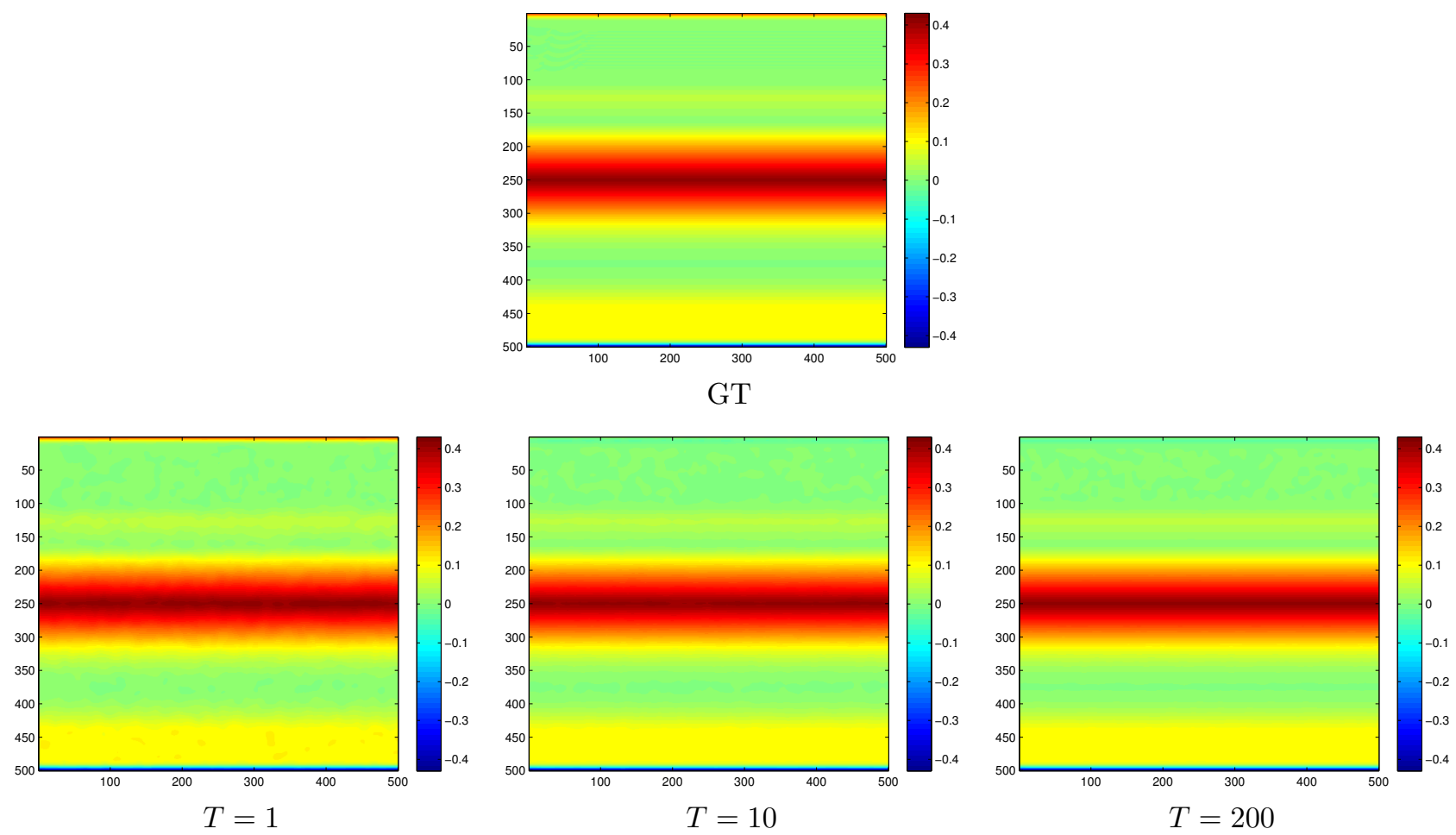

Figure 7: Method A. $\phi_{2}$
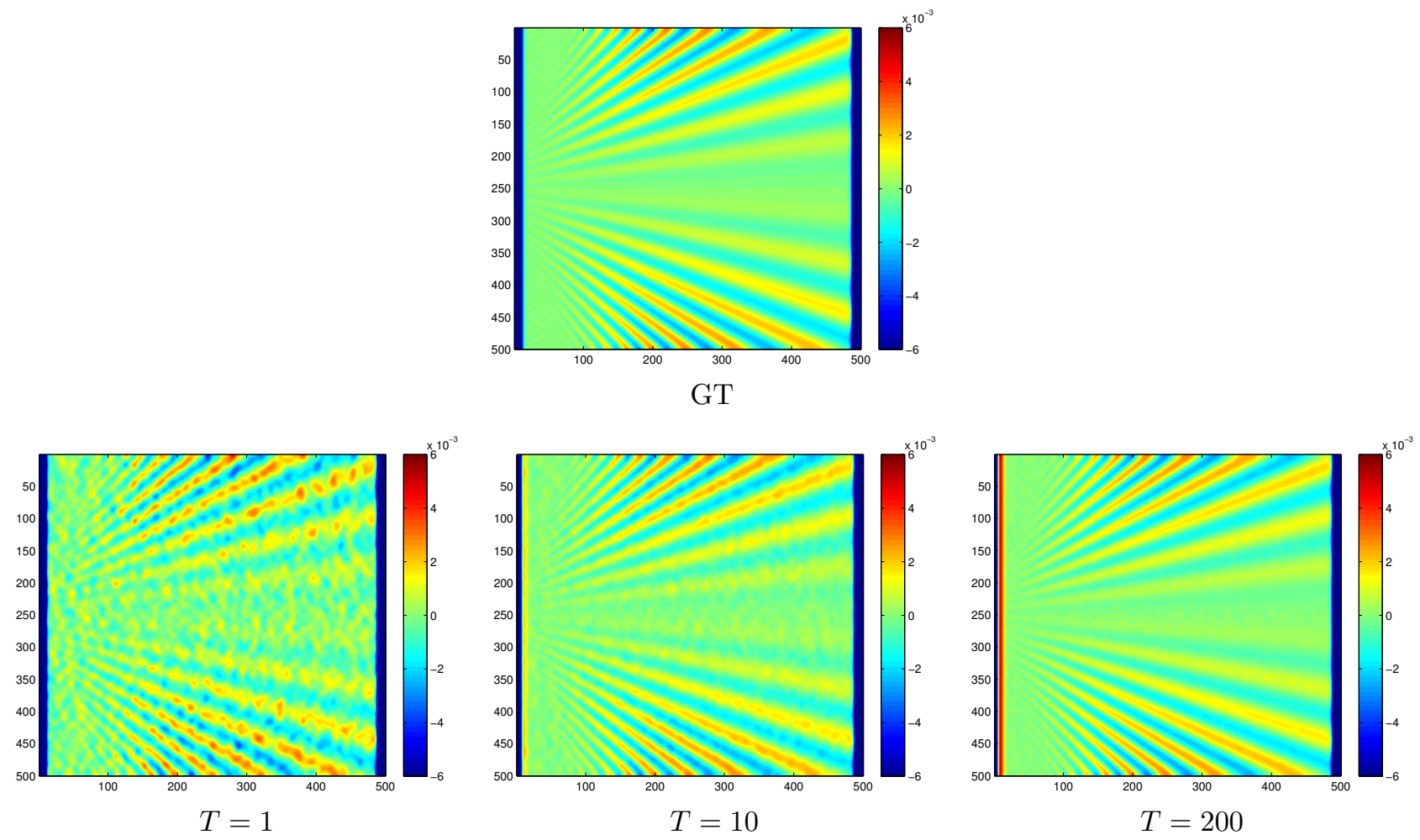

Figure 8: Method A. $\partial \phi_{1} / \partial x$ 

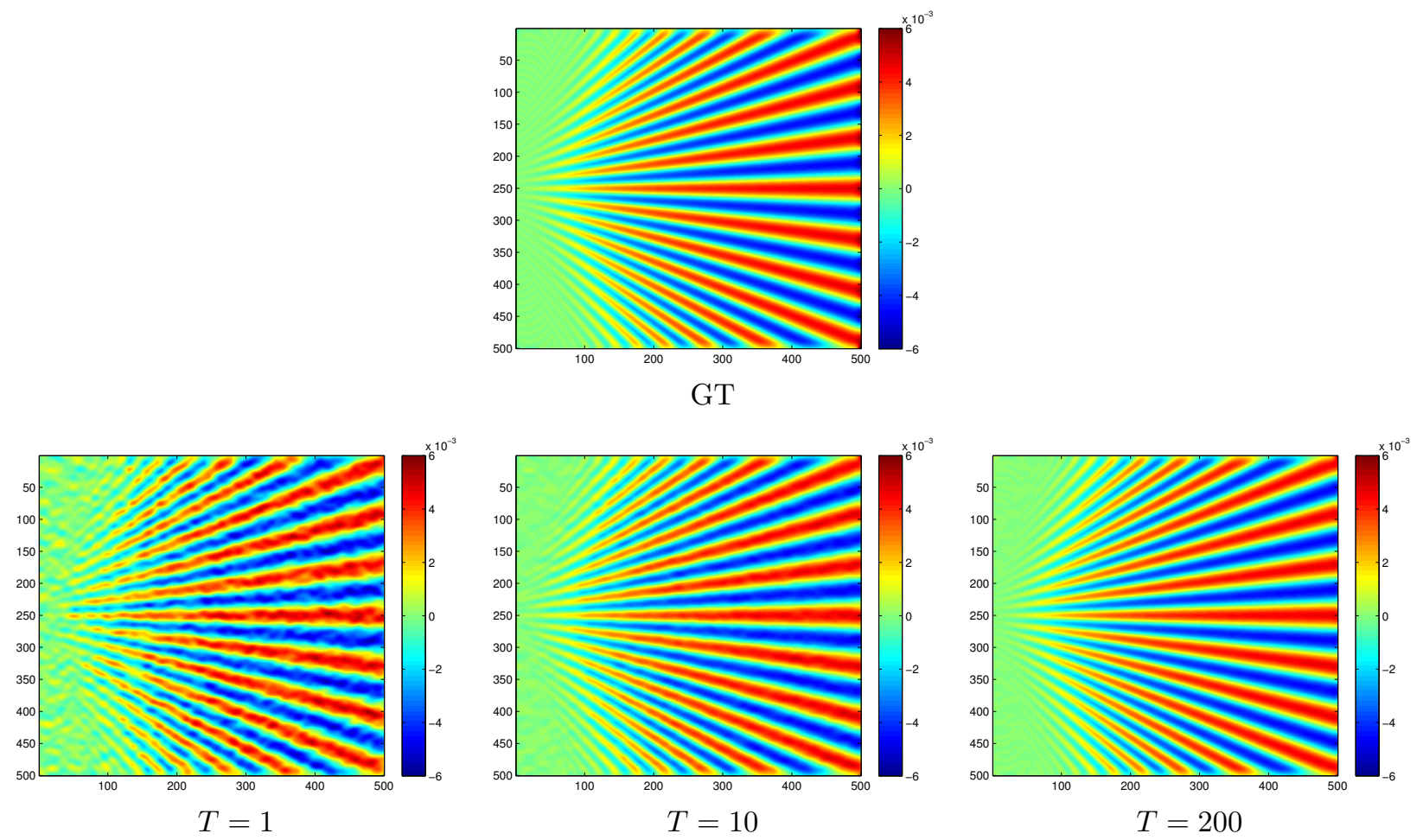

Figure 9: Method A. $\partial \phi_{1} / \partial y$
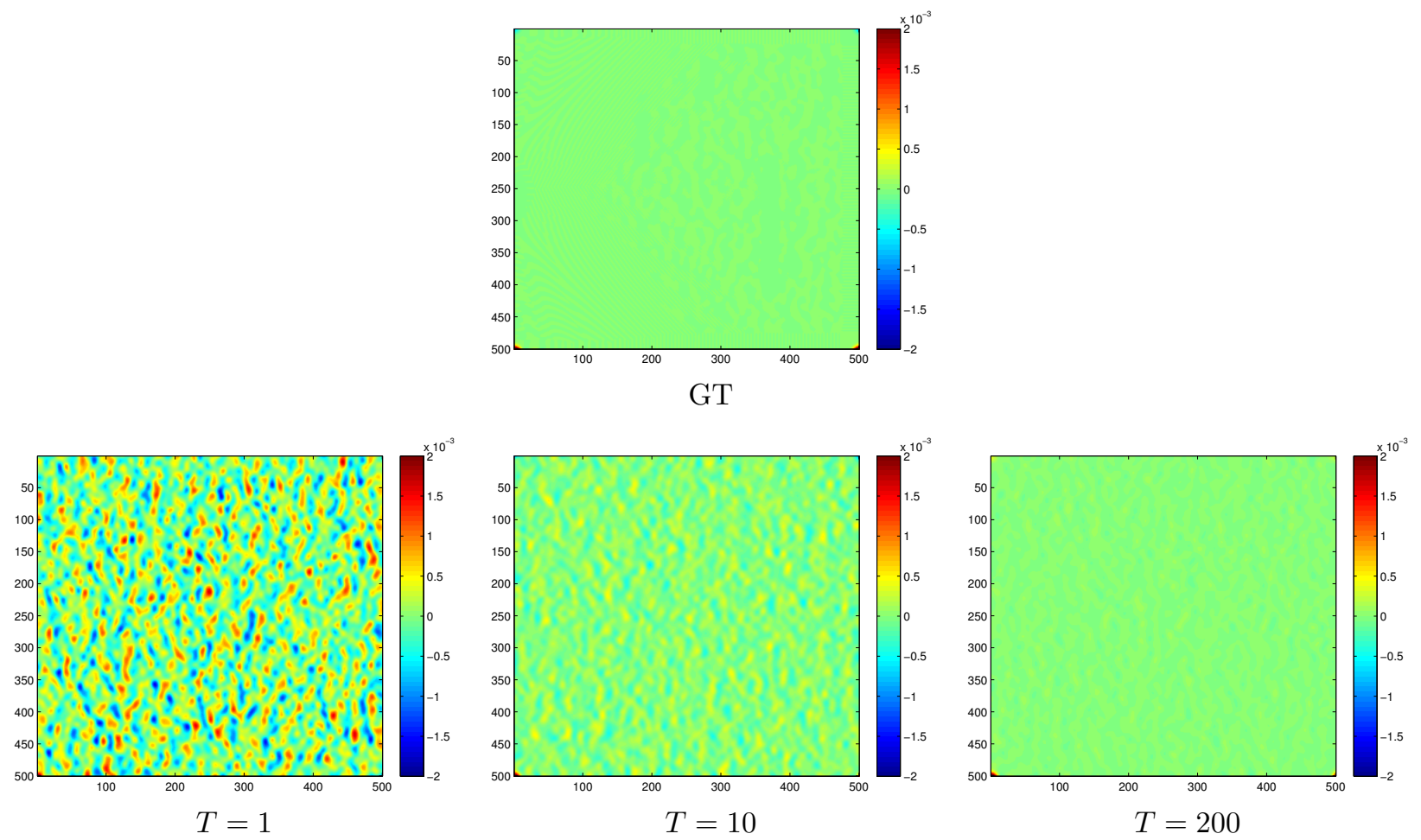

Figure 10: Method A. $\partial \phi_{2} / \partial x$. Here the ground truth corresponds to a null map. 

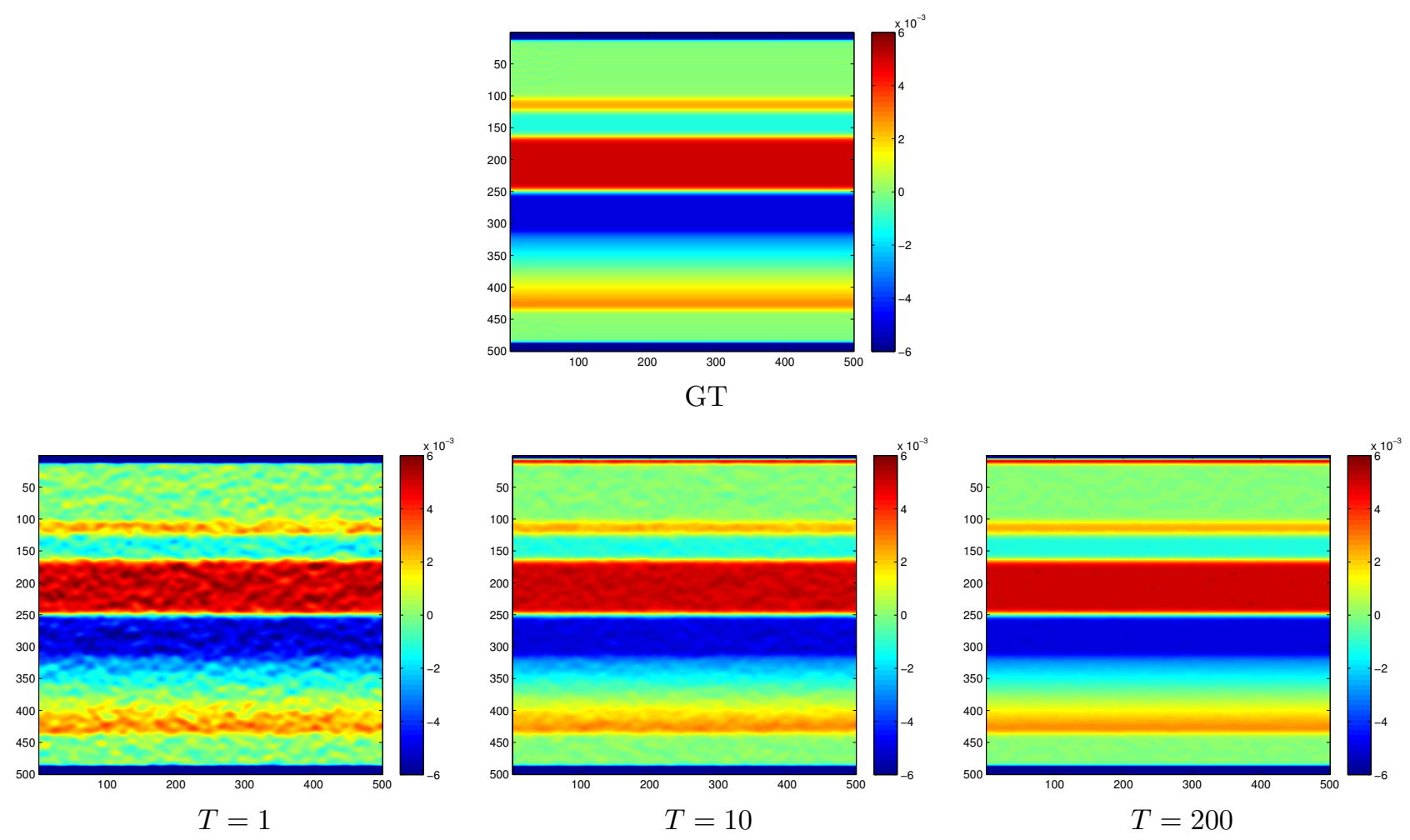

Figure 11: Method A. $\partial \phi_{2} / \partial y$ 


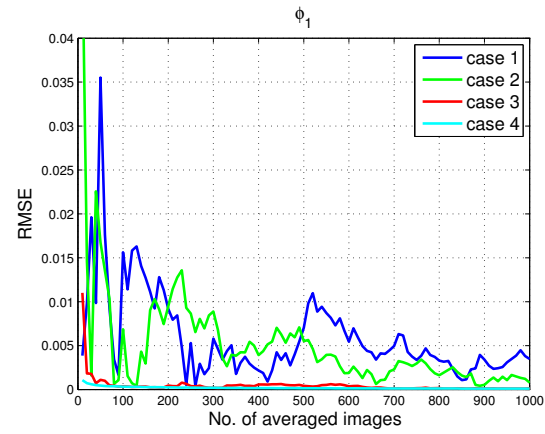

a.

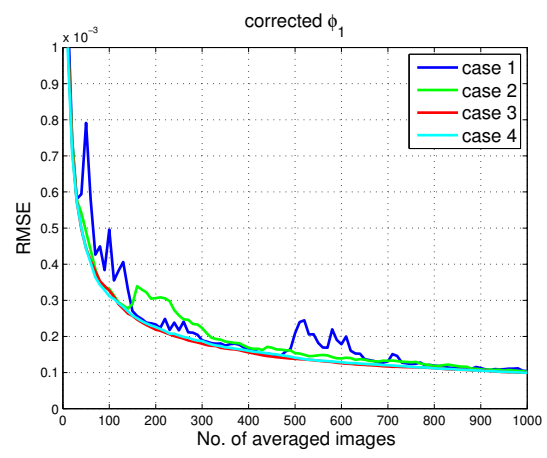

c.

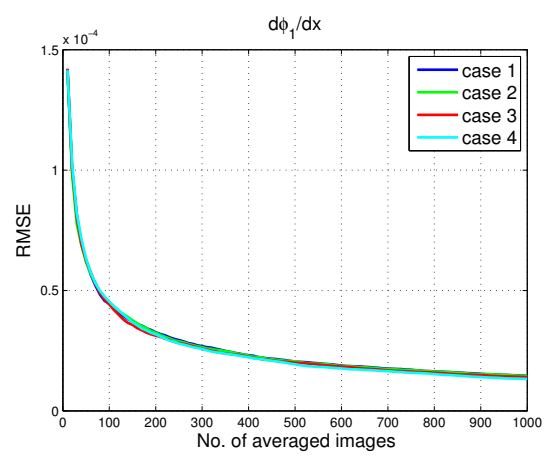

e.

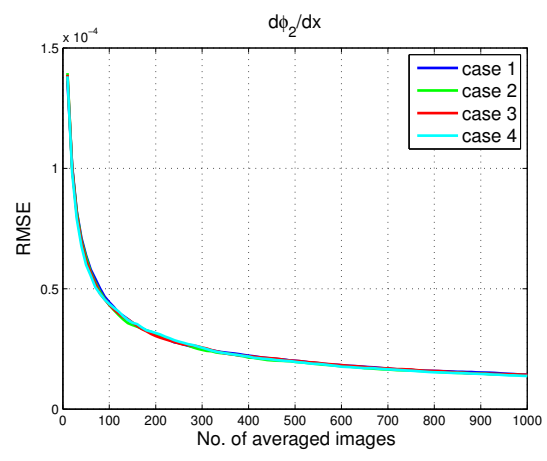

g.

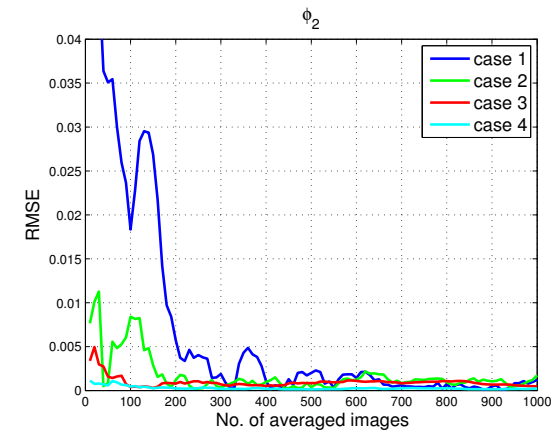

b.

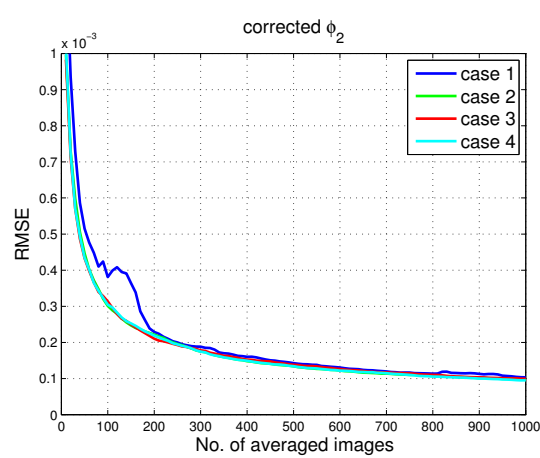

d.

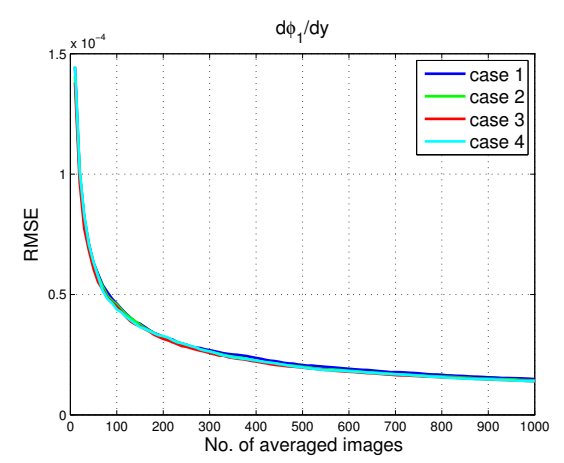

f.

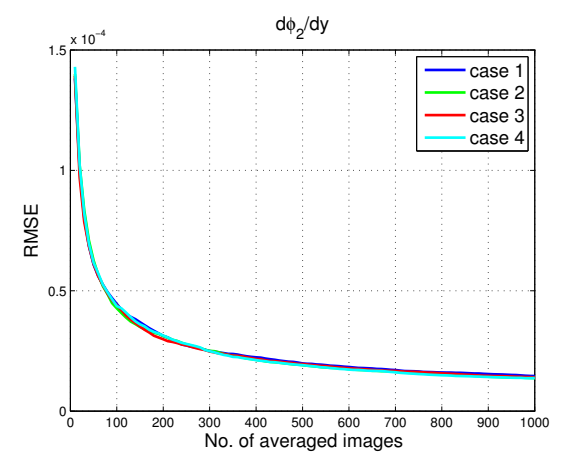

h.

Figure 12: Method A. First and second rows: RMSE for $\phi_{1}$ (a), for $\phi_{2}$ (b), for corrected $\phi_{1}$ (c), for corrected $\phi_{2}(\mathrm{~d})$. Third and four rows: RMSE for $\partial \phi_{1} / \partial x(\mathrm{e})$, for $\partial \phi_{1} / \partial y$ (f), for $\partial \phi_{2} / \partial x$ (g), for $\partial \phi_{2} / \partial y$ (h). 


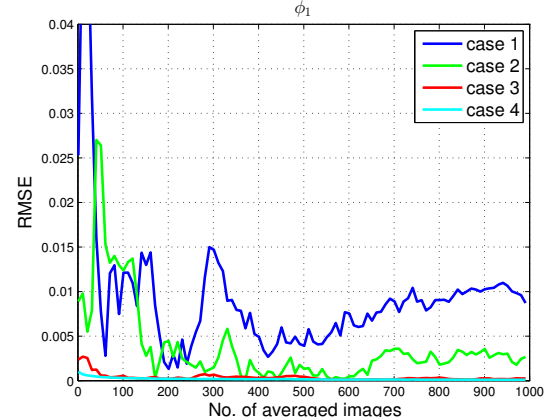

a.

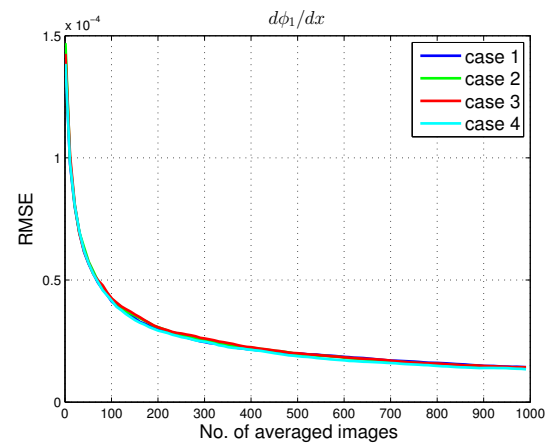

c.

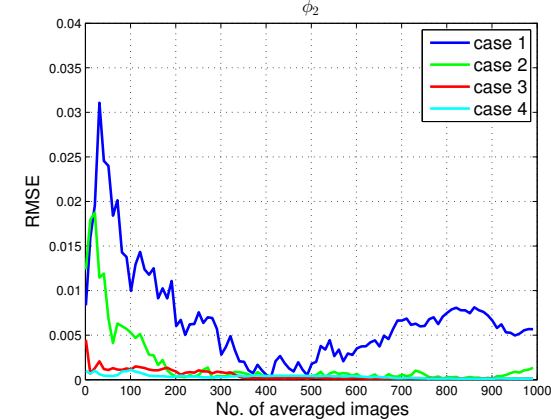

b.

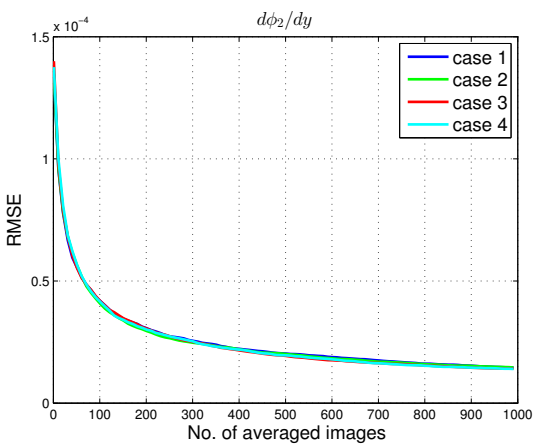

d.

Figure 13: Method B. RMSE for $\phi_{1}$ (a), for $\phi_{2}(\mathrm{~b})$, for $\partial \phi_{1} / \partial x$ (c), for $\partial \phi_{2} / \partial y$ (d). The same scales are chosen in methods $\mathbf{A}$ and $\mathbf{B}$ for comparison purpose. 


\section{Conclusion}

This paper addressed the problem of noise reduction by averaging $T$ images of a pseudo-periodic grid affected by vibrations. This problem is of the utmost importance in experimental solid mechanics since in many cases, the localized strains hardly emerge from the noise floor. It is therefore crucial to prove that the vibrations, whose effect is added to that of sensor noise in images, do not spoil the averaging process and that the measured phase and phase derivatives in the grid model (hence the displacement and strain components) are consistent unbiased estimators. We have proved on a theoretical model and experimentally assessed that the averaged grid image is a biased estimator of the unknown noise-free grid, but that the windowed Fourier transform still gives an $1 / \sqrt{T}$ unbiased estimation of the phase modulation and of its derivatives. While the phase derivatives are not affected by vibrations having an amplitude usually encountered in practice, the phases are affected by an additional component decreasing in $1 / \sqrt{T}$ which has a noticeable influence in the case of strong vibrations (standard deviation larger than 0.1 pixel in the discussion of Section 3.2). We have assessed that similar results are obtained by averaging the phases and phase derivatives obtained separately from each grid image, which is in turn much more computationally intensive.

The practical conclusion of this study is that it is legitimate to use the automatic time-averaging mode which can be found in some professional cameras such as the Sensicam QE [1] in spite of the residual vibrations, when the purpose is to extract phases and phase derivatives from the resulting averaged grid images. In the case of moderate residual vibrations (of standard deviation smaller than 0.1 pixel), numerical experiments suggest that the phase is an approximation of the ideal, vibration-free phase within a RMSE smaller than $2 \cdot 10^{-3}$ rad. Furthermore, even for larger vibrations, the phase derivatives are good approximations of the vibration-free phase derivatives within a RMSE smaller than $3 \cdot 10^{-5}$ rad.pixel $^{-1}$ for a typical stack of $T=200$ images. Besides, averaged images being biased estimators of the unknown noise-free images, it could be interesting to investigate whether measuring techniques based on the amplitude of the signal and not on the phase, such as digital image correlation [26], are affected by this bias which concerns any image (thus speckle), not only grid images. 


\section{Appendix}

We simplify the expression of $\delta_{1}$ by establishing (29) from (28)

For any complex number $z \neq 0, \arg (z)=\arctan (\operatorname{Im}(z) / \operatorname{Re}(z))$, and the arctangent addition formula writes

$$
\arctan (x)+\arctan (y)=\arctan \left(\frac{x+y}{1-x y}\right)
$$

as soon as $x y<1$.

With $x=\frac{\left(1-\frac{1}{2}(2 \pi f)^{2} \overline{\alpha^{2}}\right) \operatorname{Im}\left(d_{1}\right)+2 \pi f \bar{\alpha} \operatorname{Re}\left(d_{1}\right)}{\left(1-\frac{1}{2}(2 \pi f)^{2} \bar{\alpha}^{2}\right) \operatorname{Re}\left(d_{1}\right)-2 \pi f \bar{\alpha} \operatorname{Im}\left(d_{1}\right)}$ and $y=-\operatorname{Im}\left(d_{1}\right) / \operatorname{Re}\left(d_{1}\right)$, the condition $x y<1$ is satisfied as soon as $T$ is large enough since in this case $\bar{\alpha} \rightarrow 0$ and $x y \rightarrow-\left(\operatorname{Im}\left(d_{1}\right) / \operatorname{Re}\left(d_{1}\right)\right)^{2}$.

From (36), we successively calculate

$$
\begin{aligned}
\delta_{1} & =\arctan \left(\frac{2 \pi f \bar{\alpha} \operatorname{Re}\left(d_{1}\right)^{2}+2 \pi f \bar{\alpha} \operatorname{Im}\left(d_{1}\right)^{2}}{\left(1-\frac{1}{2}(2 \pi f)^{2} \overline{\alpha^{2}}\right) \operatorname{Re}\left(d_{1}\right)^{2}+\left(1-\frac{1}{2}(2 \pi f)^{2} \overline{\alpha^{2}}\right) \operatorname{Im}\left(d_{1}\right)^{2}}\right) \\
& =\arctan \left(\frac{2 \pi f \bar{\alpha}}{1-\frac{1}{2}(2 \pi f)^{2} \overline{\alpha^{2}}}\right)
\end{aligned}
$$

which is $(29)$. 


\section{References}

[1] Sensicam QE - 1288 datasheet. Technical report, PCO Imaging, 2005.

[2] T.M. Atanackovic and A. Guran. Theory of Elasticity for Scientists and Engineers. Springer, 2000.

[3] C. Badulescu, M. Bornert, J.-C. Dupré, S. Equis, M. Grédiac, J. Molimard, P. Picart, R. Rotinat, and V. Valle. Demodulation of spatial carrier images: performance analysis of several algorithms using a single image. Experimental Mechanics, 53(8):1357-1370, 2013.

[4] C. Badulescu, M. Grédiac, and J.-D. Mathias. Investigation of the grid method for accurate in-plane strain measurement. Measurement Science and Technology, 20(9):095102, 2009.

[5] Y. Barranger, P. Doumalin, J. C. Dupré, and A. Germaneau. Strain measurement by digital image correlation: Influence of two types of speckle patterns made from rigid or deformable marks. Strain, 48(5):357-365, 2012.

[6] M. Bornert, F. Brémand, P. Doumalin, J.-C. Dupré, M. Fazzini, M. Grédiac, F. Hild, S. Mistou, J. Molimard, J.-J. Orteu, L. Robert, Y. Surrel, P. Vacher, and B. Wattrisse. Assessment of digital image correlation measurement errors: Methodology and results. Experimental Mechanics, 49(3):353-370, 2009.

[7] J. Boulanger, C. Kervrann, P. Bouthemy, P. Elbau, J.-B. Sibarita, and J. Salamero. Patch-based nonlocal functional for denoising fluorescence microscopy image sequences. IEEE Transaction on Medical Imaging, 29(2):442-454, 2010.

[8] H. Faraji and W.J. MacLean. CCD noise removal in digital images. IEEE Transactions on Image Processing, 15(9):2676-2685, 2006.

[9] A. Foi, M. Trimeche, V. Katkovnik, and K. Egiazarian. Practical Poissonian-Gaussian noise modeling and fitting for single-image raw-data. IEEE Transactions on Image Processing, 17(10):17371754,2008 .

[10] M. Grédiac and F. Sur. Effect of sensor noise on the resolution and spatial resolution of displacement and strain maps estimated with the grid method. Strain, 50(1):1-27, 2014.

[11] M. Grédiac, F. Sur, C. Badulescu, and J.-D. Mathias. Using deconvolution to improve the metrological performance of the grid method. Optics and Lasers in Engineering, 51(6):716-734, 2013.

[12] G.E. Healey and R. Kondepudy. Radiometric CCD camera calibration and noise estimation. IEEE Transactions on Pattern Analysis and Machine Intelligence, 16(3):267-276, 1994.

[13] Q. Kemao. Two-dimensional windowed Fourier transform for fringe pattern analysis: Principles, applications and implementations. Optics and Lasers in Engineering, 45(2):304-317, 2007.

[14] M.H. Kutner, C.J. Nachtsheim, J. Neter, and W. Li. Applied linear statistical models (5th ed.). McGraw-Hill, 2008.

[15] P. Lava, S. Coppieters, Y. Wang, P. Van Houtte, and D. Debruyne. Error estimation in measuring strain fields with DIC on planar sheet metal specimens with a non-perpendicular camera alignment. Optics and Lasers in Engineering, 49(1):57-65, 2011.

[16] M. Lebrun, M. Colom, A. Buades, and J.-M. Morel. Secrets of image denoising cuisine. Acta Numerica, 21(1):475-576, 2012. 
[17] P. Milanfar. A tour of modern image filtering: New insights and methods, both practical and theoretical. IEEE Signal Processing Magazine, 30(1):106-128, 2013.

[18] F. Murthagh, J.L. Starck, and A. Bijaoui. Image restoration with noise suppression using a multiresolution support. Astronomy and astrophysics, 112:179-189, 1995.

[19] G.W. Oehlert. A note on the Delta method. The American Statistician, 46(1):27-29, 1992.

[20] F. Sur and M. Grédiac. Enhancing with deconvolution the metrological performance of the grid method for in-plane strain measurement. In Proceedings of the IEEE International Conference on Acoustics, Speech, and Signal Processing (ICASSP), pages 1563-1567, Vancouver, British Columbia, Canada, 2013.

[21] F. Sur and M. Grédiac. Measuring the noise of digital imaging sensors by stacking raw images affected by vibrations and illumination flickering. 2014. Submitted for publication.

[22] F. Sur and M. Grédiac. Sensor noise modeling by stacking pseudo-periodic grid images affected by vibrations. IEEE Signal Processing Letters, 21(4):432-436, 2014.

[23] F. Sur and M. Grédiac. Towards deconvolution to enhance the grid method for in-plane strain measurement. AIMS Inverse Problems and Imaging, 8(1):259-291, 2014.

[24] Y. Surrel. Additive noise effect in digital phase detection. Applied Optics, 36(1):271-276, 1997.

[25] Y. Surrel. Photomechanics, volume 77 of Topics in Applied Physics, chapter Fringe analysis, pages 55-102. Springer, 2000.

[26] M. Sutton, J.-J. Orteu, and H. Schreier. Image Correlation for Shape, Motion and Deformation Measurements. Springer, 2009.

[27] D. Teyssieux, S. Euphrasie, and B. Cretin. Thermal detectivity enhancement of visible and near infrared thermography by using super-resolution algorithm: Possibility to generalize the method to other domains. AIP Journal of Applied Physics, 105(6):064911-064914, 2009.

[28] S.P. Timoshenko and J. N. Goodier. Theory of elasticity. McGraw-Hill, 3rd edition edition, 1970.

[29] S.-H. Tung and M.-H. Shih. Precision verification of a simplified three-dimensional DIC method. Optics and Lasers in Engineering, 49(7):937-945, 2011. 